\title{
Management of immature non-vital tooth with single visit MTA apexification: A case report.
}

\author{
Dr. Rajshekhar Chatterjee ${ }^{1}$, Dr. Venugopal P. ${ }^{2}$, Dr. Jyothi K. N. ${ }^{3}$, \\ Dr. Jayashankar C. M. ${ }^{4}$ \\ ${ }^{I}$ (PGT, Department of Conservative Dentistry \&Endodontics, Sree Siddhartha Dental College and Hospital, \\ Karnataka, India) \\ ${ }^{2}$ (Professor, Department of Conservative Dentistry \&Endodontics, Sree Siddhartha Dental College and \\ Hospital, Karnataka, India) \\ ${ }_{3}^{3}$ HOD, Department of Conservative Dentistry \&Endodontics, Sree Siddhartha Dental College and Hospital, \\ Karnataka, India) \\ ${ }^{4}$ (Reader, Department of Conservative Dentistry \&Endodontics, Sree Siddhartha Dental College and Hospital, \\ Karnataka, India)
}

\begin{abstract}
Management of fracture dimmature teeth presents a special challenge to dentists. Apexification, Apexogenosis or Regeneration are the only options for conserving such tooth. Traditional apexification with calcium hydroxide is associated with certain difficulties, like longer treatment time, risk of tooth fracture and incomplete calcification of apical bridge. Treatment outcome of regenerations are not much convincing. Moreover, as all these treatments take longer time to show their outcome. Thus use of a biocompatible material which can form a physical apical barrier with tissue healing properties became a material of choice for treating these types of cases. Although various such materials are available, MTA has got all its favourable properties to serve as an immediate apical barrier. This case report demonstrates application of MTA apical plug followed by custom made post and core for the re-construction of maxillary central incisor. The patient was evaluated for a period of one year and peri-apical radiographs demonstrate complete healing of the peri-apical lesion. This new technique is advantageous in teeth with open apex and thin radicular dentin, where treatment can be completed in fewer visits.
\end{abstract}

\section{Introduction}

The objective of root canal treatment is to remove infection and create a favourable environment where peri-apical healing can take place. After achieving adequate disinfection a fluid tight apical seal is recommended for total obliteration of root canal space so that bacterial toxins and degraded tissue serum cannot cause periapical tissue irritation ${ }^{1}$. To achieve this fluid tight apical seal the canal obturating material has to have a confirmed apical matrix. Apical constriction of the tooth plays a great role in achieving this apical matrix. In immature teeth this natural apical constriction is not present, but as the tooth matures the apex grows and gets constricted forming its natural apical construction ${ }^{2}$. In few cases this natural maturation of root apex doesn't take place due to destruction of its pulp dentin complex by trauma or progressive infection from dental caries. Treating those cases by conventional root canal therapy possesses a challenge for the dentist, as the large open apical foramen does not provide an apical matrix for obturation. Moreover, as the tooth is immature the divergent thin dentinal root walls are very susceptible to fracture ${ }^{3}$. Above all these disadvantages, these types of cases are frequently associated with peri-apical pathologies ${ }^{2}$. Thus selection of material to treat these types of cases has to be done very consciously.

The material that is going to treat this type of cases should have few properties like, they should be bio active in nature, should be able to create a fluid tight seal, should reinforce the root dentine, and should also be anti bacterial ${ }^{4}$.Earlier various materials like calcium hydroxide powder mixed with different vehicles, collagen calcium phosphate, osteogenic protein, bone growth factor and oxidised cellulose, have been tried to achieve successful treatment but none of these materials seem to achieve their desired goal efficiently within convenient time frame $e^{3,5}$.

To overdrive this time consuming apexification technique, many alternatives have been suggested which has been aimed mainly at the development of one-step procedure (single-visit apexification). This includes creating a non-resorbable apical barrio with Mineral tri-oxide aggregate (MTA) $)^{2,3,4}$.

MTA possesses all the possible advantages over conventional apexification materials. It is very biocompatible and bio-active in nature, and promotes hard tissue healing, creates a mono block effect with the root dentine and strengthensit, it is also very anti bacterial because of its alkaline $\mathrm{Ph}^{6}$.

This case report emphasizes management of immature non-vital tooth with open apex and peri-apical lesion using single visit apexification of MTA apical plug. 


\section{Case Reports}

A 21 year male patient was referred to the Department of Conservative Dentistry and Endodontics, Sri Siddhartha Dental College, Tumkur. Patient complains of fractured upper front tooth and gave history of trauma 10 years back with no significant medical record. Clinical examination revealed Ellis class III fracture with discoloration and clogged food debris with respect to11. Mild vestibular tenderness was present in relation to 11 at the root apex and subjected tooth was mild tender on percussion.

The concerned tooth did not respond to both electric and heat test.Intraoral periapical radiograph demonstrate dradiolucentlesion with blunderbusscanal,associated periapical lesion in relation to 11. (Fig: 1).

Provisionaldiagnosed was concluded asnon-vital tooth with periapical lesion in relation to 11.Nonsurgical endodontic apexification with MTA apical plug was planned for 11 .

After proper isolation with rubber dam an appropriate access cavity was prepared to allow the debridement of the necrotic pulp. A barbed broach was used for debridement. Working length was determined2 $\mathrm{mm}$ short of the apex, in order to not injure the apical tissue (Fig: 2).Gentle circumferential filing had been performed with minimal dentin removal using \#80 H file. Saline and 2\% Chlorhexidene were used as irrigating solution. Paper point had been introduced inside canal to dry it ${ }^{7}$.

MTA was mixed with provided liquid by themanufacturer's and introduced inside the canal with a plastic filling instrument. Pre selected Pluggers were used to gentlycondense MTA into the canal ${ }^{8,9}$. After checking apical plug of MTA with radiograph, a moist cotton pellet was introduced inside the canal to hydrate the material (Fig: 3 ).

After 24hrs temporary restoration was removed and MTA was checked for setting. Then a post preparation had been performed with peeso-reamer in a sequence up to 3 in size for the preparation of custom made post space. Direct Wax pattern was made for prepared post space and it was subjected to casting procedure. Cast post was tried inside the canal, checked for occlusal clearance and cemented with type-1 Glass ionomer cement (Fig: 4).

Case has been followed in every six month and one intra oral peri apical radiograph was taken to evaluate the rate of healing for a period of one year. A clear diminishing of the lesion has been observed at the end of year itself (Fig: 5,6,).

\section{Discussion}

Obtaining a good hermatic seal in teeth with necrotic pulp and wide-open apices is a challenge in endodontics. For more than 40 years, such cases are approached clinically with apexification using $\mathrm{Ca}(\mathrm{OH})_{2}$, which acts as a initiatorfor the formation of osteoid or cementoid barrier over the apical foramen ${ }^{1,4}$. This takes month to years of follow-ups as an essential part of this type of treatment options. Moreover wash out effect of $\mathrm{Ca}(\mathrm{OH})_{2}$ makes this treatment modality amultiple-visit approach. Previous studies have described the disadvantages of calcium hydroxide apexification ${ }^{2,4}$. Obturation with tailor-made gutta-percha, termed as Roll cone technique has also been proposed as an alternative to $\mathrm{Ca}(\mathrm{OH})_{2}$ apexification. However, the difficulty to achieve hermetic seal, has led to discontinuing this type of treatment options ${ }^{1,4,10}$

Revascularization which is very conservative approach,is considered as a good alternative treatment option for these types of cases. However, there are no randomized controlled clinical trials available till date for the success of theseprocedures on teeth with persistent peri-apical infection. Moreover in the present case the subjected tooth was indicated for post and core, where root canal space was required for placement of intra radicular post ${ }^{11}$.

Considering all this we have decided to perform apexification using MTA plug, which will allow immediate apical closure, promotes apical healing and strengthens the root dentin.

MTA has come up as a very good alternative in treating these types of cases, where the material sets and creates an apical barrier readily. It has got a very good sealing ability, as the material immediately bonds with the roots and creates a mono block. Its high Ph helps to destroy the surrounding microorganisms and its bio active nature stimulates blastic cells to create favourable environment for healing ${ }^{4,5,6}$. It also promotes cementum deposition on it ${ }^{12}$.Moreover, due to its fast setting time, fewer follow-up appointments are required to carry out this treatment.

Not only the selection of material, but also the thickness of apical MTA barrier has played a key role in clinical success. A $5 \mathrm{~mm}$ thick apical MTA barrier has proven to be significantly stronger with lesser leakage than a $2 \mathrm{~mm}$ thick barrier. In the present case since the root canal space was required for post and core, inspite of making $3 \mathrm{~mm}$ apical plug a condensed $5 \mathrm{~mm}$ apical MTA plug was chosen ${ }^{10,13}$.

\section{Conclusion}

Single step apexification with a novel biocompatible and bio-active material like MTA is a convenient approach for managing immature open apex roots. Although this procedure seems to be a little expensive than conventional approach, but if we consider the long term success and patient's convenience, that extra 
economical burden will not be of much problem. Long term follow-ups ( $<2$ years) are still awaited to justify the long term clinical success of this procedure.

\section{Acknowledgement}

I acknowledge DR. K. R Kashinath for his support and co-operation

\section{References}

[1] Jhon I Ingle, Leif K Bakland, J Craig Baumgartner. Ingle’s Endodontics. Sixth edition. India: CBS; 2013.

[2] Kenneth M. Hargreaves, Stephen Cohen. Cohen's pathway of the pulp. Tenth edition. India: Elsevier; 2011.

[3] Beenaphilipmathew ,mithra n. Hegde. Management of non vital immature teeth - cases reports and review. Endodontology. 2010:18-22

[4] Shikhadogra ,Mukundaks, ArunaA,Shwetha m rao. Apexification. Journal of dental sciences and research. 2012; 3(1): 41-44

[5] Donald R, James O, CtnilYesilsoy. Apexification: review of the literature. Quintsence International 1990;7: 589-98

[6] Don Schmitt, Jacob Lee, George Bogen. Multifaceted use of ProRoot MTA root canal repair material. American Academy of Pediatric Dentistry. 23:4, 2001: 326-30.

[7] S. Simon, F. Rilliard, A. Berdal, P. Machtou. The use of mineral trioxide aggregate in one-visit apexification treatment: a prospective study. International Endodontic Journal 2007; 40: 186-97

[8] Girish. C. Kubasad, Sheetal.B. Ghivari. Apexification with apical plug of MTA- report of cases. AOSR 2011; 1(2):104-107.

[9] AntonisChaniotis, Greece. Open-apex retreatment under the operating microscope. Roots 2010; 4:1-4

[10] Anil Kumar G, Kavitha A. Single Visit Apexification with Mineral Trioxide Aggregate. IJDA 2010; 2(1): 122-24

[11] Todd M, Geisler. Clinical considerations for regenerative endodontic procedure. DCNA 2012; 56: 603-26

[12] Pedro Felicio Estrada et al. Comparative study of MTA and other materials inretrofilling of pulpless dogs' teeth. Braz Dent J 2005; 16(2): 149-155

[13] Ahmed Al-Kahtani, Sandra Shostad, Robert Schifferle, SatishBhambhani.In-vitro evaluation of microleakage of an orthogradeapical plug of mineral trioxide aggregate in permanentteeth with simulated immature apices.JEndod 2005; 31(2) :117119
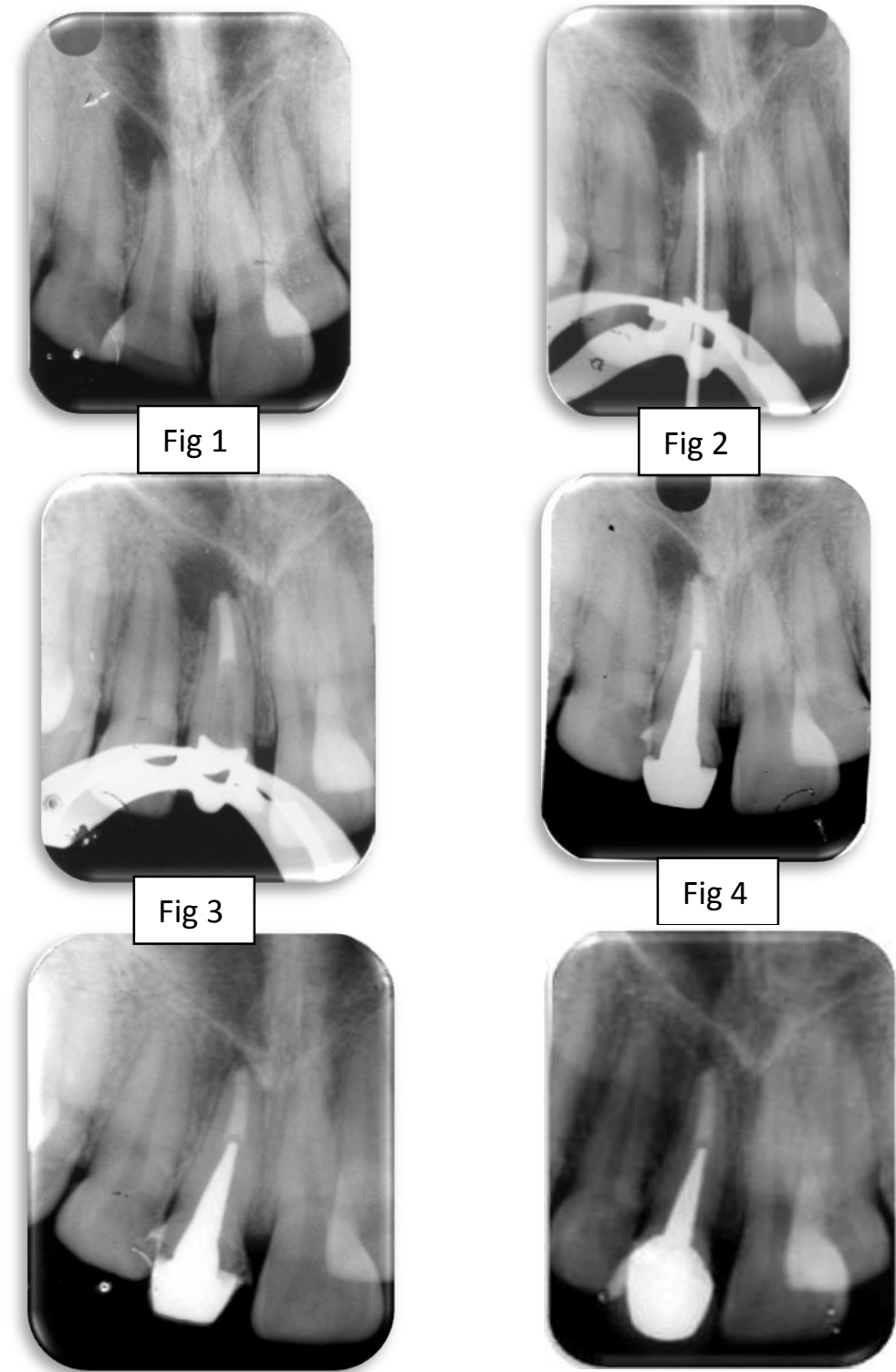